\title{
Understanding the mechanisms of nuclear collisions: A complete study of the ${ }^{10} \mathrm{~B}+{ }^{120} \mathrm{Sn}$ reaction
}

\author{
L. R. Gasques $\odot,{ }^{1,}{ }^{*}$ M. A. G. Alvarez $\odot,{ }^{2}$ A. Arazi $\odot,{ }^{3}$ B. V. Carlson, ${ }^{4}$ L. C. Chamon $\odot,{ }^{1}$ J. P. Fernández-García $\odot,{ }^{2}$ \\ A. Lépine-Szily $\odot,{ }^{1}$ J. Lubian $\odot,{ }^{5}$ J. Rangel, ${ }^{5}$ M. Rodríguez-Gallardo, ${ }^{2,6}$ V. Scarduelli, ${ }^{1}$ and V. A. B. Zagatto ${ }^{5}$ \\ ${ }^{1}$ Universidade de São Paulo, Instituto de Fisica, Rua do Matao, 1371, 05508-090 São Paulo, São Paulo, Brazil \\ ${ }^{2}$ Departamento de Física Atómica, Molecular y Nuclear, Universidad de Sevilla, Apartado 1065, 41080 Sevilla, Spain \\ ${ }^{3}$ Laboratorio TANDAR, Comisión Nacional de Energía Atómica, Avenida General Paz 1499, BKNA1650 San Martín, Argentina \\ ${ }^{4}$ Departamento de Física, Instituto Tecnológico de Aeronáutica, Centro Técnico Aeroespacial, São José dos Campos, São Paulo, Brazil \\ ${ }^{5}$ Instituto de Física da Universidade Federal Fluminense, 24210-346 Niterói, Rio de Janeiro, Brazil \\ ${ }^{6}$ Instituto Carlos I de Física Teórica y Computacional, Universidad de Sevilla, Spain
}

(Received 25 November 2020; accepted 8 March 2021; published 22 March 2021)

\begin{abstract}
Background: Reactions involving exotic and stable weakly bound nuclei have been extensively studied in recent years. Although several models have been successfully used to explain particular reaction outcomes, the answers to many questions remain elusive. In previous works, we presented elastic, inelastic, and transfer angular distributions for the ${ }^{10} \mathrm{~B}+{ }^{120} \mathrm{Sn}$ system measured at $E_{\mathrm{Lab}}=31.5,33.0,35.0$, and $37.5 \mathrm{MeV}$. The data set was analyzed through coupled reaction channels calculations in the context of the double-folding São Paulo potential.

Purpose: We investigate nuclear reaction mechanisms for systems involving weakly bound projectiles.

Method: Angular distributions for several nuclear reaction processes were measured for the ${ }^{10} \mathrm{~B}+{ }^{120} \mathrm{Sn}$ system at $E_{\text {Lab }}=39.70 \mathrm{MeV}$.

Results: The new data set involves angular distributions for elastic scattering, projectile and target inelastic excitations, one-neutron pickup transfer, one-proton stripping transfer, deuteron pickup transfer, and ${ }^{3,4} \mathrm{He}$ stripping transfer. We have also observed ${ }^{10} \mathrm{Be}$ nuclei. The effect of the couplings to some nonelastic states on the angular distributions is discussed.

Conclusion: The theoretical calculations within the coupled reaction channels formalism provide an overall good agreement with the corresponding inelastic, one-neutron stripping, one-proton pickup, one-deuteron pickup, and ${ }^{3} \mathrm{He}$ stripping transfer data. However, to improve the description of the elastic scattering angular distribution, the inclusion of additional channels in the coupling scheme might be necessary.
\end{abstract}

DOI: 10.1103/PhysRevC.103.034616

\section{INTRODUCTION}

The production of isotopes far from the valley of stability has paved a new road in science for exploring fundamental aspects of the complex interaction of a quantum many body system involving nuclei with exotic neutron to proton ratios. However, even after many important technical developments, these species are still extremely difficult to produce in the laboratory. In contrast, most of the existing stable nuclei can be produced with standard techniques in copious amounts. Consequently, the investigation of heavy-ion reactions involving weakly bound stable nuclei represents one of the leading research subjects in low energy nuclear physics, and is an important tool to explore the properties of radioactive nuclei. Over many years, a significant number of different nuclear processes have been experimentally determined, revealing valuable information to guide the development of new theoretical models [1-8].

*Corresponding author: lgasques@if.usp.br
In previous experimental campaigns carried out at the Institute of Physics of the University of São Paulo (USP), using the $8 \mathrm{MV}$ Pelletron accelerator installed in the Open Laboratory of Nuclear Physics (LAFN, acronym in Portuguese), we have measured angular distributions of elastic scattering, inelastic excitations, and transfer processes at energies spanning the Coulomb barrier for several systems involving weakly bound projectiles [9-11]. The data have been analyzed within the coupled reaction channel (CRC) formalism using the well known double-folding São Paulo potential (SPP) [12] for the nuclear interaction. In particular, we also investigated the ${ }^{10} \mathrm{~B}+{ }^{120} \mathrm{Sn}$ system at energies below and slightly above the barrier $\left(V_{B} \simeq 35 \mathrm{MeV}\right.$ in the laboratory reference system): $E_{\mathrm{Lab}}=31.5,33.5,35$, and $37.5 \mathrm{MeV}$. For this system, besides the CRC, we also performed continuumdiscretized coupled-channels (CDCC) calculations. ${ }^{10} \mathrm{~B}$ is a weakly bound stable nucleus that may breakup into different partitions, the most energetically favorable being ${ }^{10} \mathrm{~B} \rightarrow$ ${ }^{6} \mathrm{Li}+{ }^{4} \mathrm{He}(Q=-4.461 \mathrm{MeV})$. As reported in Ref. [11], the effect of the couplings to the continuum on the elastic scattering cross sections was almost negligible for all those energies. It is worth mentioning that we did not consider the excitation 
of the ${ }^{120} \mathrm{Sn}$ target in these $\mathrm{CDCC}$ calculations. Recently, we also studied the reaction ${ }^{10} \mathrm{~B}+{ }^{197} \mathrm{Au}$. As part of a joint collaboration among research groups from Argentina, Brazil, Costa Rica, and Spain, the experiments were performed at two laboratories: LAFN and TANDAR (Tandem of Argentina), which is equipped with a $20 \mathrm{MV}$ tandem accelerator. Elastic scattering, inelastic excitations, and transfer and fusion processes have been experimentally determined at several energies [13,14], below and well above the Coulomb barrier $\left(V_{B} \simeq 50 \mathrm{MeV}\right)$, up to $E_{\mathrm{Lab}}=61 \mathrm{MeV}$.

In the present paper, we move forward with the investigation of the ${ }^{10} \mathrm{~B}+{ }^{120} \mathrm{Sn}$ system, reporting on new measurements at $E_{\mathrm{Lab}}=39.70 \mathrm{MeV}$. At this energy, which is about $15 \%$ above the barrier height, we observed more reaction processes than were detected in the lower energy region [11]. In fact, apart from the elastic scattering channel, several processes were experimentally observed at $E_{\mathrm{Lab}}=$ $39.70 \mathrm{MeV}$, such as inelastic excitation of several ${ }^{10} \mathrm{~B}$ and ${ }^{120} \mathrm{Sn}$ states, one-neutron $(1 n)$ pickup transfer, one-proton (1p) stripping transfer, deuteron pickup transfer, and ${ }^{3,4} \mathrm{He}$ stripping transfer. We have also observed ${ }^{10} \mathrm{Be}$ yields, which may have been produced by two different mechanisms: (i) the direct single-charge exchange (SCE), related to the isovector nucleon-nucleon interaction, and (ii) a two-step process, in which the incident ${ }^{10} \mathrm{~B}$ can undergo two possible sequential transfer reactions, in which a proton and a neutron are exchanged: ${ }^{120} \mathrm{Sn}\left({ }^{10} \mathrm{~B},{ }^{11} \mathrm{~B}\right){ }^{119} \mathrm{Sn}$ followed by ${ }^{119} \mathrm{Sn}\left({ }^{11} \mathrm{~B},{ }^{10} \mathrm{Be}\right){ }^{120} \mathrm{Sb}$, and ${ }^{120} \mathrm{Sn}\left({ }^{10} \mathrm{~B},{ }^{9} \mathrm{Be}\right){ }^{121} \mathrm{Sb}$ followed by ${ }^{121} \mathrm{Sb}\left({ }^{9} \mathrm{Be},{ }^{10} \mathrm{Be}\right){ }^{120} \mathrm{Sb}$.

The paper is organized as follows: details of the experimental setup and data analysis are discussed in Sec. II. The experimental results and theoretical CRC analyses are presented in Sec. III. A summary of the paper and the main conclusions are presented in Sec. IV.

\section{EXPERIMENTAL SETUP AND DATA ANALYSIS}

The experiment was carried out at the 30B scattering chamber of LAFN of USP, São Paulo, Brazil,which is equipped with an $8 \mathrm{MV}$ tandem accelerator. The incident beam was produced by extracting atomic ions from an enriched ${ }^{10} \mathrm{~B}$ isotope cathode mounted in a 32-position MC-SNICS ion source. After passing throughout the tank, the post-accelerated beam interacted with a ${ }^{120} \mathrm{Sn}$ target with thickness of about $300 \mu \mathrm{g} / \mathrm{cm}^{2}$, evaporated on to $15 \mu \mathrm{g} / \mathrm{cm}^{2}$ carbon backing. For normalization, a thin layer of ${ }^{197} \mathrm{Au}$ $\left(\approx 50 \mu \mathrm{g} / \mathrm{cm}^{2}\right)$ was evaporated over the ${ }^{120} \mathrm{Sn}$ film. Taking into account the average energy loss of the ${ }^{10} \mathrm{~B}$ incident particles in the middle of the target, the angular distributions were obtained at $E_{\mathrm{Lab}} \approx 39.70 \mathrm{MeV}$. As depicted in Fig. 1, our detection system is composed of two different particle detector arrays: SATURN (Silicon Array \& Telescopes of USP for Nuclear Reactions and Nuclear Applications) and STAR (Silicon Telescopes Array for low statistics nuclear Reactions). These arrays can be mounted in different configurations according to the necessity of each experiment.

In the measurements presented in this paper, the SATURN array comprised a set of nine single semiconductor silicon surface barrier detectors mounted at the backward hemisphere,

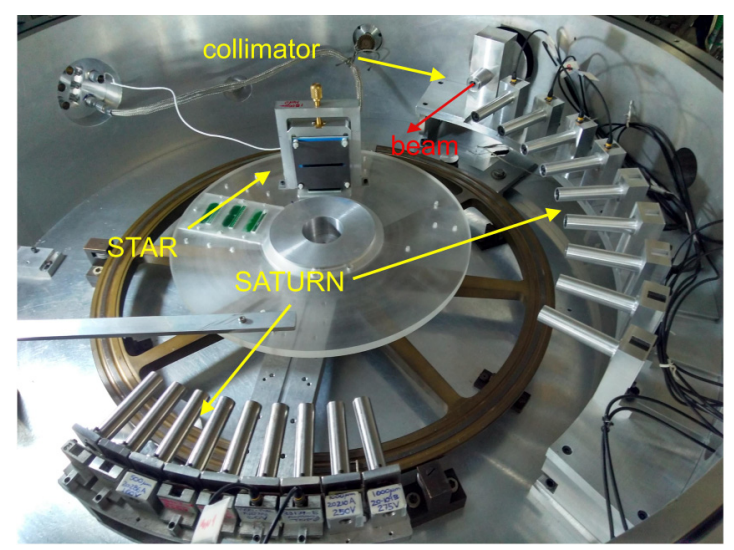

FIG. 1. Internal view of the scattering chamber at the 30B beam line of LAFN.

giving information about the kinetic energy of the particles. For normalization of the cross sections, other two surface barrier detectors were mounted at $50^{\circ}$ and $60^{\circ}$. At such forward angles, the elastic scattering cross sections are the same as the Rutherford ones for both systems: ${ }^{10} \mathrm{~B}+{ }^{120} \mathrm{Sn}$ and ${ }^{10} \mathrm{~B}+{ }^{197} \mathrm{Au}$. A typical spectrum obtained with a single detector mounted at $\theta_{\mathrm{Lab}}=170^{\circ}$ in the SATURN array is shown in Fig. 2. Several peaks are identified and labeled in the figure using different colors (see the figure caption).

In the present experiment, the STAR array was mounted with only one $(\Delta E, E)$ telescope, covering an angular range of about $40^{\circ}$. The first detection stage $(\Delta E)$ was formed by a $20 \mu \mathrm{m}$ thick single sided silicon strip detector (SSSSD) with 16 strips. The second detector, with thickness of about $1000 \mu \mathrm{m}\left(E_{r}\right)$, was coupled to the $\Delta E$ detector. Both detectors were manufactured by Micron Semiconductor. Particle identification was performed based on the energy loss measurements of $\Delta E$ and residual energy $E_{r}$.

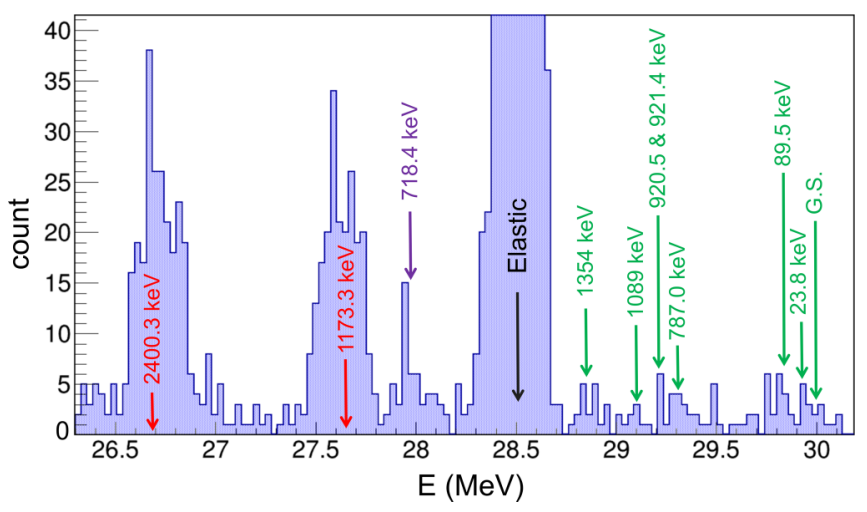

FIG. 2. Single-channel spectrum taken with a detector at $\theta_{\text {Lab }}=$ $170^{\circ}$ mounted in the SATURN array. The arrows indicate the expected positions of peaks related to the elastic scattering of ${ }^{10} \mathrm{~B}$ on ${ }^{120} \mathrm{Sn}$ target (black arrow), inelastic excitation of the $1^{+}{ }^{10} \mathrm{~B}$ first excited state at $718.4 \mathrm{keV}$ (purple), and excitation of the $2^{+}(1173.3 \mathrm{keV})$ and $3^{-}(2400.3 \mathrm{keV}){ }^{120} \mathrm{Sn}$ states (red). The peaks corresponding to different states of the $1 n$ pickup transfer ${ }^{120} \mathrm{Sn}\left({ }^{10} \mathrm{~B},{ }^{11} \mathrm{~B}\right){ }^{119} \mathrm{Sn}$ are indicated by green arrows. 


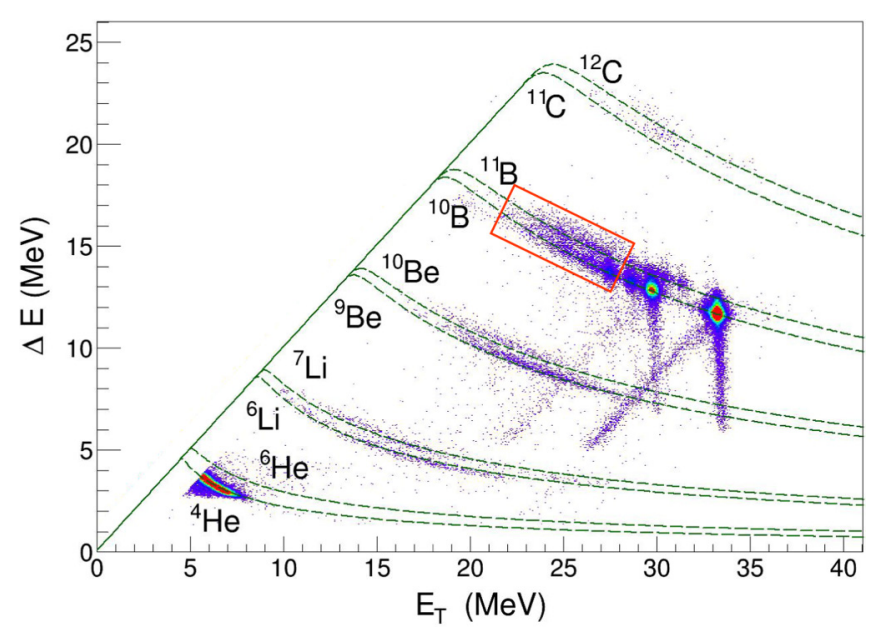

FIG. 3. $\left(\Delta E, E_{T}\right)$ spectrum taken with the STAR array at $\theta_{\mathrm{Lab}}=$ $128.6^{\circ}$. The dashed lines correspond to theoretical calculations of energy loss in the $\Delta E$ detector for several identified nuclei produced during the collision of ${ }^{10} \mathrm{~B}$ on the target composed of ${ }^{120} \mathrm{Sn}$ and ${ }^{197} \mathrm{Au}$. The rectangular area contains events of ${ }^{11} \mathrm{~B}$ (from neutron transfer) and ${ }^{10} \mathrm{~B}$ (from inelastic scattering to states with high excitation energies).

Figure 3 presents a typical two-dimensional $\left(\Delta E, E_{T}\right)$ spectrum obtained at $\theta_{\mathrm{Lab}}=128.6^{\circ}$, where $E_{T}=\Delta E+E_{r}$. Taking into account the energy loss of the different particles traversing the $\Delta E$ detector, it was possible to identify each nucleus located in the respective bands. The dashed lines in the figure represent theoretical calculations for the energy loss in the $\Delta E$ detector as a function of the total energy $E_{T}$ of different nuclei. In the $Z=5$ band, the regions containing the highest counting rates correspond to the ${ }^{10} \mathrm{~B}$ elastically scattered on ${ }^{120} \mathrm{Sn}$ and ${ }^{197} \mathrm{Au}$. Events corresponding to the inelastic scattering of ${ }^{10} \mathrm{~B}+{ }^{120} \mathrm{Sn}$ and ${ }^{10} \mathrm{~B}+{ }^{197} \mathrm{Au}$ can also be observed in the same band. They lie in the left side of the corresponding elastic scattering spots. The ${ }^{11} \mathrm{~B}$ yields, arising from the $1 n$ pickup transfer reactions with ${ }^{120} \mathrm{Sn}$ and ${ }^{197} \mathrm{Au}$, can also be observed in the spectrum. The ${ }^{120} \mathrm{Sn}\left({ }^{10} \mathrm{~B},{ }^{11} \mathrm{~B}\right){ }^{119} \mathrm{Sn}$ and ${ }^{197} \mathrm{Au}\left({ }^{10} \mathrm{~B},{ }^{11} \mathrm{~B}\right){ }^{196} \mathrm{Au}$ reactions have, respectively, positive $Q$ values of about 2.35 and $3.38 \mathrm{MeV}$ for their ground states (g.s.). Therefore, some ${ }^{11} \mathrm{~B}$ events can be found on the right side (higher energies) of the elastic scattering, but the detected ${ }^{11} \mathrm{~B}$ extend up to a region of high excitation energies (with boundaries represented through solid lines in the figure).

Different regions, corresponding to $Z=2,3,4,5$, and 6 , are clearly observed in Fig. 3. Considering that ${ }^{10} \mathrm{~B}$ can be described as a cluster of ${ }^{6} \mathrm{Li}+{ }^{4} \mathrm{He}$, the yields of both nuclei arising from the breakup channel should be quite similar. However, an inspection at the $Z=2$ and 3 regions of Fig. 3 shows that ${ }^{4} \mathrm{He}$ are produced in excess, as compared with ${ }^{6} \mathrm{Li}$. A possible explanation lies on the fact that ${ }^{4} \mathrm{He}$ nuclei are produced in copious amounts through the fusion of ${ }^{10} \mathrm{~B}$ projectiles impinging on ${ }^{12} \mathrm{C}$ present in the target. The incident energy $\left(E_{\mathrm{Lab}}=39.70 \mathrm{MeV}\right)$ is much higher than the Coulomb barrier for the ${ }^{10} \mathrm{~B}+{ }^{12} \mathrm{C}$ reaction. Another possible explanation for the excess of alpha particles would be the

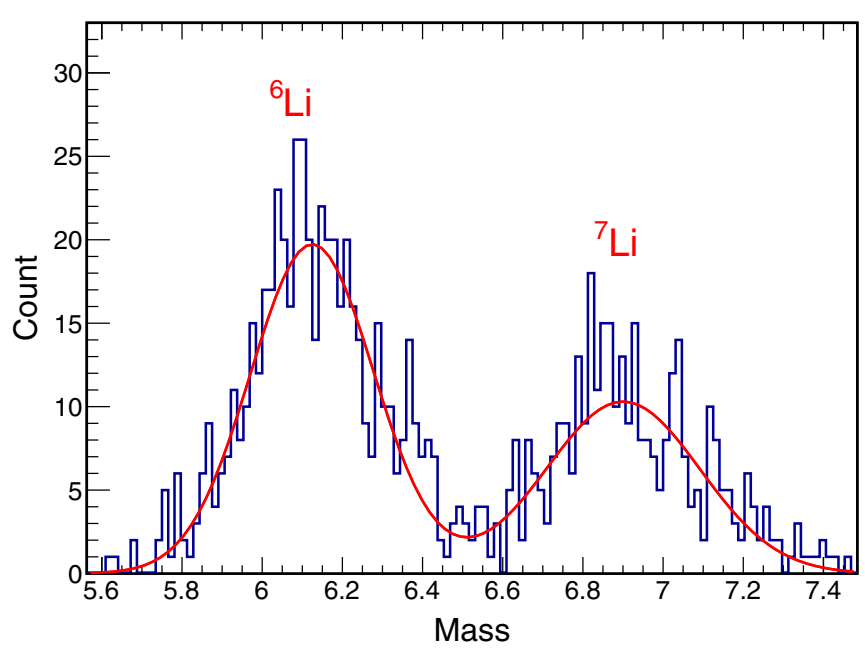

FIG. 4. Number of events related to the lithium isotopes projected on the mass axis, obtained from the $\left(\Delta E, E_{T}\right)$ spectrum of Fig. 3 using the method described in Ref. [15].

breakup of ${ }^{6} \mathrm{Li}$ into an alpha and deuteron after the breakup of ${ }^{10} \mathrm{~B}$. Nevertheless, this is a second step process and might be not very relevant at this bombarding energy. Also, other breakup or transfer reaction channels with positive $Q$ values may produce alpha particles as well.

As is well known, the mass separation between different isotopes is rather difficult in $\left(\Delta E, E_{T}\right)$ spectra, since the dependence of the energy loss of the particles on the mass $m$ is weaker than the dependence on $Z$. Aiming to improve the resolution of the mass separation among particles with the same $Z$, a method of data processing described in Ref. [15] has been applied here. The main attribute of the method consists of the linearization of the data on the $m$ axis using theoretical calculations of energy loss of the particles. In this paper, the masses of the nuclei are given in atomic mass units (amu). As can be observed in Fig. 4, a good separation of ${ }^{6} \mathrm{Li}$ and ${ }^{7} \mathrm{Li}$ has been achieved in our experiment. A fit of the ${ }^{6} \mathrm{Li}$ $\left({ }^{7} \mathrm{Li}\right)$ events considering a Gaussian shape results in peak positions at $6.12 \pm 0.06(6.90 \pm 0.09)$, which are compatible with $m=6(m=7)$. In fact, these small deviations of the peaks related to the mass are expected, since the method of Ref. [15] is based on the energy loss calculated for the average mass (in this case, $m=6.5$ ). Figure 5 presents the projection of the ${ }^{9} \mathrm{Be}$ and ${ }^{10} \mathrm{Be}$ events on the $m$ axis. Clearly, the mass resolution of the peaks becomes gradually worse as the $Z$ of the particles increases. Even so, the separation of ${ }^{9} \mathrm{Be}$ from ${ }^{10} \mathrm{Be}$ is still quite good.

In the case of the $Z=5$ band, the very intense peak of the ${ }^{10} \mathrm{~B}$ elastic scattering becomes impracticable to separate from the quite small yields of events relative to ${ }^{11} \mathrm{~B}$ detected with energies around those of the elastically scattered ${ }^{10} \mathrm{~B}$. Even so, it is possible to distinguish ${ }^{11} \mathrm{~B}$ and ${ }^{10} \mathrm{~B}$ in other energy regions far from the elastic scattering. Figure 6 presents the projection on the mass axis of boron events restricted to the $\left(\Delta E, E_{T}\right)$ region with boundaries shown by the rectangular area in Fig. 3. 


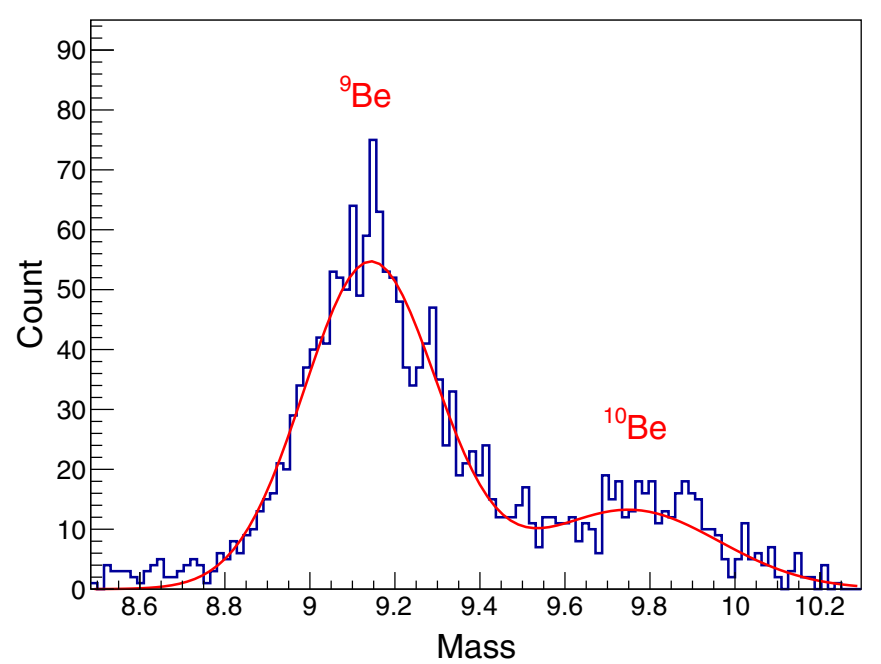

FIG. 5. The same as Fig. 4, for the beryllium isotopes.

\section{EXPERIMENTAL AND THEORETICAL RESULTS}

Experimental elastic scattering angular distributions were obtained using both SATURN and STAR arrays. Concerning inelastic excitation, yields corresponding to the $1_{1}^{+}\left(E^{*}=\right.$ $0.72 \mathrm{MeV}){ }^{10} \mathrm{~B}$ quadrupole state, and to the $2_{1}^{+}(1.17 \mathrm{MeV})$ quadrupole and the $3_{1}^{-}(2.40 \mathrm{MeV})$ octupole ${ }^{120} \mathrm{Sn}$ vibrational states have been experimentally determined. In addition, events still corresponding to ${ }^{10} \mathrm{~B}$ were detected with the STAR array in a relatively broad region of higher excitation energies $\left(2.5 \leqslant E^{*} \leqslant 4.5 \mathrm{MeV}\right)$. The experimental cross section for these events is related to the excitation of the projectile and/or target.

Differential cross sections for the $1 n$ pickup, $1 p$ stripping, and deuteron pickup transfer were determined through the corresponding yields observed in the spectra. Cross sections related to the production of ${ }^{6} \mathrm{Li},{ }^{7} \mathrm{Li}$, and ${ }^{10} \mathrm{Be}$, that can be

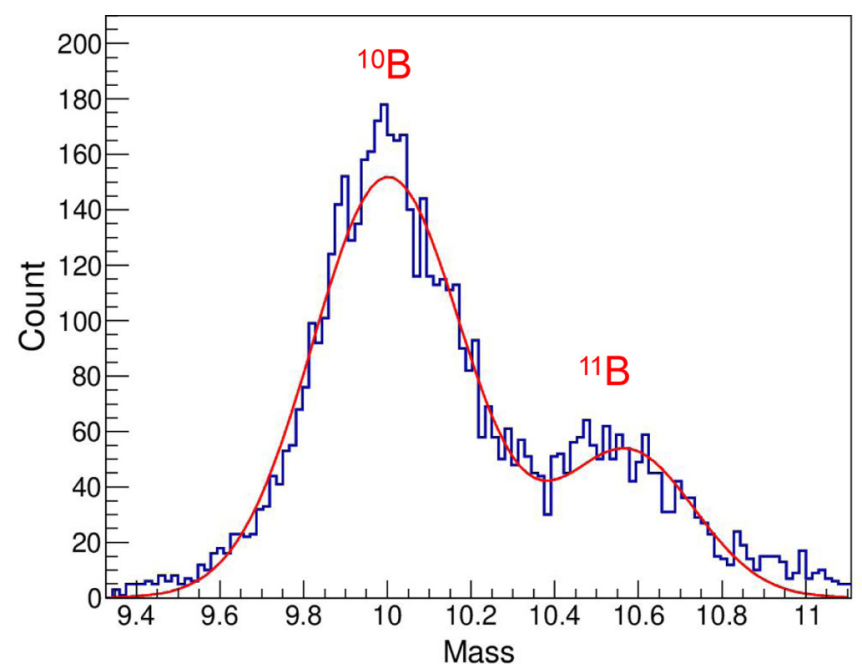

FIG. 6. Number of events related to the boron isotopes projected on the mass axis, obtained for events inside the $\left(\Delta E, E_{T}\right)$ region delimited by solid lines in Fig. 3. associated to breakup processes, were also determined in the experiment.

CRC calculations were carried out assuming the SPP for the real part of the nuclear interaction [12]. We adopted a phenomenological Woods-Saxon (WS) parametrization for the imaginary part of the optical potential. Since several peripheral channels were explicitly considered in the coupling scheme, the parameters adopted for the imaginary potential, $W_{0}=80 \mathrm{MeV}, r_{i 0}=0.8 \mathrm{fm}$, and $a_{i}=0.30 \mathrm{fm}$, were set to account only for the absorption of flux following barrier penetration, resulting in negligible strength in the surface region. In this condition, the theoretical cross sections are insensitive to reasonable variations of the imaginary potential parameter values.

The CRC calculations were performed using the FRESCO code [16]. In the coupling scheme, we have included the first $1_{1}^{+}$excited state of ${ }^{10} \mathrm{~B}$, and the ${ }^{120} \mathrm{Sn}$ vibrational states corresponding to the $2_{1}^{+}$quadrupole and the $3_{1}^{-}$octupole. Since the ${ }^{10} \mathrm{~B}$ projectile is highly deformed, we have adopted the same procedure as described in Ref. [10], where the ${ }^{10} \mathrm{~B}$ was considered as a rotor and as a vibrator. As will be shown in the paper, although the global theoretical results are similar, the assumption of a rotational quadrupole state for ${ }^{10} \mathrm{~B}$ provides a slightly better description of the elastic and inelastic experimental data. As ${ }^{10} \mathrm{~B}$ has a $3^{+}$g.s., we have also investigated the effect of considering a real spin-orbit (s.o.) term in the central part of the optical potential. We have adopted the same parameter values applied in the case of the ${ }^{10} \mathrm{~B}+{ }^{197} \mathrm{Au}$ system [14]: $V_{0}=6 \mathrm{MeV}, r_{0}=1.06 \mathrm{fm}$, and $a=0.6 \mathrm{fm}$.

As already commented, yields related to the inelastic excitation of nearby populated states of the projectile and target in the excitation energy region of $2.5 \leqslant E^{*} \leqslant 4.5 \mathrm{MeV}$ have been experimentally observed. A realistic calculation treating explicitly all the many excited states present in this energy range is certainly impracticable. Therefore, in the CRC calculations, we adopted a schematic representation to simulate the effect of these states, which just assumes the coupling to a single ${ }^{120} \mathrm{Sn}$ level at $E^{*}=3.5 \mathrm{MeV}$. The values of spin and deformation of such a state have been varied in order to describe the experimental cross sections.

In addition to the inelastic states, we have also considered the couplings to five states related to the $1 n$ pickup transfer, ${ }^{120} \mathrm{Sn}\left({ }^{10} \mathrm{~B},{ }^{11} \mathrm{~B}\right){ }^{119} \mathrm{Sn}$, corresponding to the g.s. and a few excited states of the ${ }^{119} \mathrm{Sn}$, and two states related to the $1 p$ stripping transfer ${ }^{120} \mathrm{Sn}\left({ }^{10} \mathrm{~B},{ }^{9} \mathrm{Be}\right){ }^{121} \mathrm{Sb}$. With the purpose of simulating the effect of a large number of states, the procedure of assuming the coupling to only one state, with coupling parameter values adjusted to fit the data, was also adopted in the case of some transfer channels, as described herein.

\section{A. Elastic and inelastic angular distributions}

The experimental elastic scattering angular distribution measured at $E_{\mathrm{Lab}}=39.70 \mathrm{MeV}$ is presented in Fig. 7. As adopted in several other figures, the black circles and green squares represent cross sections obtained with the SATURN array and STAR telescope, respectively. In panel (a) of Fig. 7, it is possible to observe the results of the full (including all inelastic and transfer couplings) CRC calculations, which 


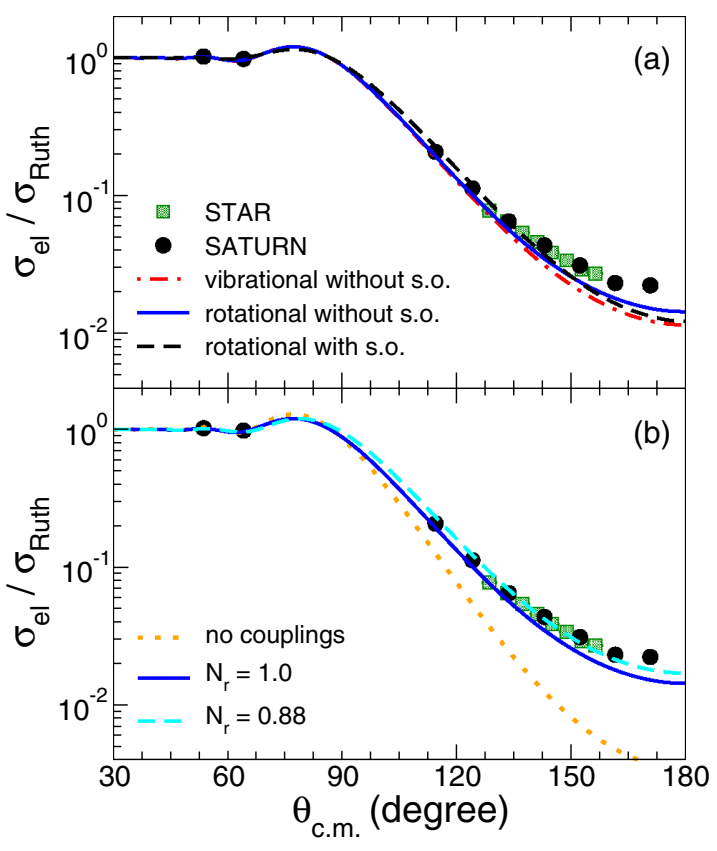

FIG. 7. Experimental cross sections for the elastic scattering of ${ }^{10} \mathrm{~B}+{ }^{120} \mathrm{Sn}$ at $39.70 \mathrm{MeV}$ bombarding energy. Theoretical results are represented by different lines (see text for details).

have been obtained by (i) considering the ${ }^{10} \mathrm{~B}$ nucleus to be a rotor with no s.o. potential (solid blue curve), or (ii) assuming the vibrational model for the ${ }^{10} \mathrm{~B}$ nucleus and no s.o. potential (dashed-dotted red curve). Both assumptions result in similar elastic scattering cross sections, although the rotational model provides marginally better agreement with the data. Actually, the reduced chi-square value obtained with the rotational model is 98 , whereas the vibrational model gives a value of 214. These somewhat large numbers are due to the quite small uncertainties of the elastic scattering data, which vary from $1 \%$ to $3 \%$. In addition, a dashed black line is also shown in panel (a) of Fig. 7. This curve was obtained by considering the s.o. potential and the rotational model for ${ }^{10} \mathrm{~B}$ in the CRC calculations. A comparison of the blue and black curves clearly shows that the effect of the s.o. interaction on the elastic scattering angular distribution is quite small. In any case, it is possible to assert that while the addition of the s.o. term slightly improves the agreement with the data in a region of intermediate scattering angles, it results in a slightly worse description of the data for $\theta_{\text {c.m. }} \gtrsim 150^{\circ}$. In order to verify the effect of the couplings on the elastic channel, the theoretical cross sections obtained with the single channel optical model (no couplings) are represented in panel (b) of Fig. 7 by the dotted orange line. As can be observed, although the effect of the couplings on the elastic channel is strong, the cross sections obtained with the full CRC calculations (solid blue curve) slightly underpredict the data. This discrepancy, which becomes more relevant as the scattering angle increases, could be related to possible couplings not included in our CRC calculations. It is possible to improve the description of the data by considering a renormalization of the nuclear interaction. In order to obtain a good data fit it is necessary to multiply the real part of the
SPP by a factor 0.88 [see the dashed cyan line in panel (b) of Fig. 7]. On the other hand, the discrepancy commented above could be related with couplings not included in our CRC calculations. This possibility is discussed in Sec. IV.

In the case of the inelastic transitions, we have considered the reduced electromagnetic transition probability:

$$
B\left(E \lambda, J_{i} \rightarrow J_{f}\right)=\frac{1}{2 J_{i}+1}\left|\left\langle J_{f} \| E \lambda|| J_{i}\right\rangle\right|,
$$

where $\lambda$ is the multipolarity of the excitation, $J_{f}$ and $J_{i}$ are, respectively, the spins of the final and initial states associated with the transition matrix element for the $E \lambda$ operator. According to Refs. $[17,18]$, we have determined the nuclear deformation $\delta_{\lambda}$ value from the respective Coulomb transition probability, considering the effect of the finite diffuseness value of the nuclear density.

The $B(E \lambda) \uparrow$ values were adjusted to fit the inelastic scattering data. To ensure a satisfactory description of the experimental inelastic scattering angular distributions, we had to assume slightly different values for the $B(E 2)$ and $B(E 3)$ deformation parameters of ${ }^{120} \mathrm{Sn}$, while treating ${ }^{10} \mathrm{~B}$ as a rotor or as a vibrator. Since the ${ }^{10} \mathrm{~B}$ is strongly deformed, its inelastic coupling affects the cross sections of the other channels through the coupled-channels formalism. Particularly for the case in which ${ }^{10} \mathrm{~B}$ is assumed to be a rotor, we have also investigated the effect of considering a s.o. term in the central potential. To obtain a satisfactory description of the inelastic data, a further readjustment of the $B(E 2)$ and $B(E 3){ }^{120} \mathrm{Sn}$ deformation parameters was required.

The spin of the inelastic states considered in the coupling scheme, along with the corresponding transition mode, excitation energy, Coulomb transition probability and deformation length are given in Table I. The values obtained here for the $B\left(E 2,0^{+} \rightarrow 2^{+}\right)$of ${ }^{120} \mathrm{Sn}$ and the $B\left(E 2,3^{+} \rightarrow 1^{+}\right)$of ${ }^{10} \mathrm{~B}$ are consistent with the respective values that we presented earlier in Ref. [10]. For the excitation of the ${ }^{120} \mathrm{Sn} 3^{-}$state, the E3 octupole transition values available in the literature show large discrepancies and large uncertainties. In the present work, in order to describe the experimental data, we found a $B(E 3)$ value which is lower than the average of values given in the literature [19-23].

The inelastic scattering angular distributions are presented in Fig. 8. Again the solid blue and dashed-dotted red lines represent, respectively, the CRC results obtained by assuming the rotational or vibrational model for ${ }^{10} \mathrm{~B}$, without a s.o. potential. The dashed black line corresponds to CRC calculations when the s.o. term is considered in the central potential. In this case, the ${ }^{10} \mathrm{~B}$ has been treated as a rotor. Panel (c) of Fig. 8 presents the inelastic scattering angular distribution associated with the relatively broad energy range of excitations of the projectile and target $\left(2.5 \leqslant E^{*} \leqslant 4.5 \mathrm{MeV}\right)$. As already mentioned, the corresponding theoretical cross sections were obtained within a simplified coupling scheme, in which a single ${ }^{120} \mathrm{Sn}$ state with $E^{*}=3.5 \mathrm{MeV}$ was adopted to simulate the effect of all possible nearby populated excited states of the projectile and target. The corresponding coupling parameters are also presented in Table I, and resulted in a satisfactory description of the data [see panel (c) of Fig. 8]. Although it is clear that the total angle integrated inelastic cross sections 
TABLE I. Spin, excitation energies (MeV), transition amplitudes from the g.s. to the excited states $\left(10^{-3} e^{2} \mathrm{~b}^{\lambda}\right)$, and deformation lengths $(\mathrm{fm})$, for the inelastic states included in the $\mathrm{CRC}$ calculations. The values given in the fifth and sixth columns are for the situation in which ${ }^{10} \mathrm{~B}$ is treated as a rotor, without the s.o. term in the central potential. The seventh and eighth columns were obtained in the rotational case, but this time including the s.o. The ninth and tenth correspond to the case in which ${ }^{10} \mathrm{~B}$ is considered as a vibrator, without the s.o. potential.

\begin{tabular}{lccccccrr}
\hline \hline Nuclues & Spin & $E^{*}$ & $\lambda$ & $\begin{array}{c}B(E \lambda) \uparrow \\
\text { rotor, no }\end{array}$ & $\begin{array}{c}\delta_{\lambda} \\
\text { s.o. }\end{array}$ & $\begin{array}{c}B(E \lambda) \uparrow \\
\text { rotor, with }\end{array}$ & $\begin{array}{c}\delta_{\lambda} \\
\text { s.o. }\end{array}$ & $\begin{array}{c}B(E \lambda) \uparrow \\
\text { vibrator, no }\end{array}$ \\
\hline${ }^{10} \mathrm{~B}$ & $1^{+}$ & 0.72 & 2 & 0.35 & 0.95 & 0.31 & 0.85 & 0.35 \\
${ }^{120} \mathrm{Sn}$ & $2^{+}$ & 1.17 & 2 & 230 & 0.68 & 194 & 0.62 & 250 \\
${ }^{120} \mathrm{Sn}$ & $3^{-}$ & 2.40 & 3 & 58 & 0.62 & 48 & 0.56 & 0.95 \\
${ }^{120} \mathrm{Sn}$ & $2^{+}$ & 3.50 & 2 & 230 & 0.68 & 202 & 0.64 & 260 \\
\hline \hline
\end{tabular}

depend on the adopted model, the overall fit to the data is quite good in all the considered cases. Similar behavior also occurs in the cases of the transfer channels. Therefore, from now on we will present the CRC cross sections obtained assuming only the model in which ${ }^{10} \mathrm{~B}$ is a rotor, without the inclusion of the s.o. potential.

\section{B. Transfer processes}

The transfer processes in heavy-ion experiments are generally highly selective as the description of their corresponding cross sections is governed by the kinematic conditions of the reactions. Part of this feature can be understood in terms of the $Q$ value of the reaction, which is often compared to another quantity called the optimum $Q$ value, $Q_{\text {opt }}$, obtained from the matching conditions of the semiclassical trajectory [24]:

$$
Q_{\mathrm{opt}}=E_{\mathrm{c} . \mathrm{m} .}\left(\frac{Z_{P}^{f} Z_{T}^{f}}{Z_{P}^{i} Z_{T}^{i}}-1\right)
$$

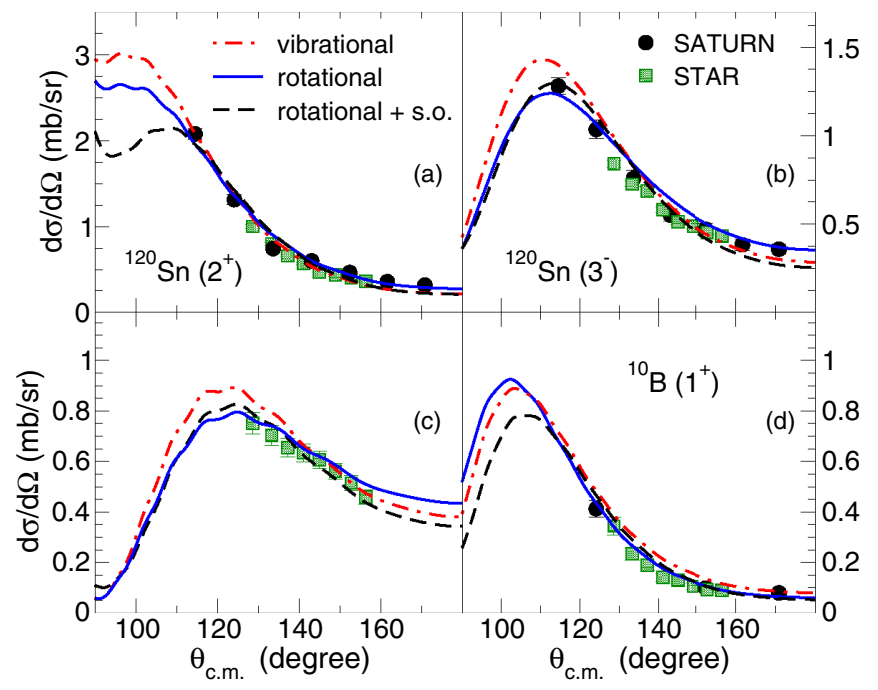

FIG. 8. Inelastic scattering angular distributions for the (a) $2^{+}$ quadrupole and (b) $3^{-}$octupole ${ }^{120} \mathrm{Sn}$ states. The cross sections corresponding to the inelastic states in the energy region of $2.5 \leqslant$ $E^{*} \leqslant 4.5 \mathrm{MeV}$ are presented in panel (c). The inelastic scattering angular distribution for the $1^{+}{ }^{10} \mathrm{~B}$ state is given in panel (d). The lines represent the theoretical results of the CRC calculations (see text for details).
In Eq. (2), $E_{\text {c.m. }}$ is the center-of-mass energy and $Z_{P}^{f}, Z_{T}^{f}, Z_{P}^{i}, Z_{T}^{i}$ are the atomic numbers of the nuclei in the final and initial partitions, respectively. Often, the value of $Q_{\text {opt }}$ is used to give an estimation of the energy region where the transfer cross section is expected to be largest. Table II shows the $Q_{\text {opt }}$ and $Q_{g s}$ values for some transfer processes, where $Q_{g s}$ is the $Q$ value for the transfer to the g.s. of the final products. For the cases where $Q_{g s}$ is larger than $Q_{\text {opt }}$, the transfer would preferentially populate states of the final nuclei with excitation energies around $E_{\text {Theo }}^{*}=Q_{g s}-Q_{\mathrm{opt}}$. In contrast, if $Q_{g s}<Q_{\text {opt }}$, the transfer would populate states close to the g.s., i.e., $E_{\text {Theo }}^{*}=0$, and it could be rather inhibited. Table II also presents the values of $E_{\mathrm{Theo}}^{*}$ and $E_{\mathrm{Exp}}^{*}$, where $E_{\mathrm{Exp}}^{*}$ is the average excitation energy related to the respective transfer process obtained from our experimental spectra. Due to the presence of the intense neighbor peak of elastic scattering, it was not possible to determine $E_{\operatorname{Exp}}^{*}$ for the neutron transfer. Indeed, events related to this channel were observed in a wide region of excitation energy, starting from the g.s. (see Fig. 3).

In the CRC calculations, a WS shape with parameter values presented in Table III was assumed for the particle-core potentials of the transfer reactions. These parameters were adjusted to reproduce the SPP results at the surface region.

\section{One-neutron transfer}

The experimental angular distributions for $1 n$ pickup were obtained from the yields of three groups related to different states of the residual nuclei. The first group includes the g.s. and the first three ${ }^{119} \mathrm{Sn}$ excited states. The second group contains many states in the range of excitation energy varying from $E^{*}=0.80 \mathrm{MeV}$ to $E^{*}=2.10 \mathrm{MeV}$. Detected ${ }^{11} \mathrm{~B}$ nuclei

TABLE II. $Q_{g s}, Q_{\mathrm{opt}}, E_{\text {Theo }}^{*}$, and $E_{\mathrm{Exp}}^{*}$ values for some transfer processes in the ${ }^{10} \mathrm{~B}+{ }^{120} \mathrm{Sn}$ reaction. The quantities are provided in units of $\mathrm{MeV}$.

\begin{tabular}{lcccr}
\hline \hline Exit channel & $Q_{g s}$ & $Q_{\text {opt }}$ & $E_{\text {Theo }}^{*}$ & $E_{\text {Exp }}^{*}$ \\
\hline${ }^{11} \mathrm{~B}+{ }^{119} \mathrm{Sn}$ & 2.35 & 0 & 2.35 & \\
${ }^{12} \mathrm{C}+{ }^{118} \mathrm{In}$ & 8.18 & 6.47 & 1.7 & 4.1 \\
${ }^{11} \mathrm{C}+{ }^{119} \mathrm{In}$ & -2.00 & 6.47 & 0 & \\
${ }^{10} \mathrm{Be}+{ }^{120} \mathrm{Sb}$ & -3.24 & & & 5.5 \\
${ }^{9} \mathrm{Be}+{ }^{121} \mathrm{Sb}$ & -0.80 & -6.77 & 6.0 & 7.1 \\
${ }^{7} \mathrm{Li}+{ }^{123} \mathrm{Te}$ & -4.78 & -13.83 & 9.0 & 12.2 \\
${ }^{6} \mathrm{Li}+{ }^{124} \mathrm{Te}$ & -2.61 & -13.83 & 11.2 & 12.4 \\
\hline \hline
\end{tabular}


TABLE III. Parameter values of the WS potentials assumed for the cluster-core systems in the CRC transfer calculations. The $V_{0}$ values presented in the table provide the right results for the binding energy when the compound system is in its ground state.

\begin{tabular}{lccc}
\hline \hline $\begin{array}{l}\text { Composite } \\
\text { system }\end{array}$ & $\begin{array}{c}V_{0} \\
(\mathrm{MeV})\end{array}$ & $\begin{array}{c}R_{0} \\
(\mathrm{fm})\end{array}$ & $\begin{array}{c}a \\
(\mathrm{fm})\end{array}$ \\
\hline$n+{ }^{10} \mathrm{~B}$ & 46.4 & 3.00 & 0.61 \\
$n+{ }^{119} \mathrm{Sn}$ & 48.7 & 6.16 & 0.65 \\
$p+{ }^{9} \mathrm{Be}$ & 41.8 & 3.00 & 0.61 \\
$p+{ }^{120} \mathrm{Sn}$ & 131 & 6.16 & 0.65 \\
$d+{ }^{10} \mathrm{~B}$ & 41.8 & 3.00 & 0.75 \\
$d+{ }^{118} \mathrm{In}$ & 43.4 & 6.30 & 0.70 \\
${ }^{3} \mathrm{He}+{ }^{7} \mathrm{Li}$ & 136 & 2.90 & 0.62 \\
${ }^{3} \mathrm{He}+{ }^{120} \mathrm{Sn}$ & 31.2 & 6.30 & 0.66 \\
\hline \hline
\end{tabular}

related to these two groups have energies higher than that corresponding to the elastically scattered ${ }^{10} \mathrm{~B}$ and, therefore, the corresponding yields can be easily and accurately obtained from both single and $\left(\Delta E, E_{T}\right)$ spectra. The third group also contains many states, but this time in a higher region of excitation energies: $3.4 \leqslant E^{*} \leqslant 9.2 \mathrm{MeV}$. In this case, the data could be obtained only from the $\left(\Delta E, E_{T}\right)$ spectra. The depths of the WS potentials presented in Table III were adjusted to reproduce the experimental separation energies for one neutron in ${ }^{10} \mathrm{~B}\left(S_{n}=8.44 \mathrm{MeV}\right)$ and ${ }^{119} \mathrm{Sn}\left(S_{n}=6.17 \mathrm{MeV}\right)$. The corresponding values of $V_{0}$ adjusted for the g.s. of $\left(n+{ }^{10} \mathrm{~B}\right)$ and $\left(n+{ }^{119} \mathrm{Sn}\right)$ are given in Table III. Similar $V_{0}$ values were obtained for the excited states. The spectroscopic factors $\left(C^{2} S_{\ell j}\right)$ associated with the g.s. transition and with the first three excited states of ${ }^{119} \mathrm{Sn}$ are given in Table IV. We emphasize that these values were obtained in data fits performed in our earlier work at lower bombarding energies [10].

Panel (a) of Fig. 9 presents experimental and theoretical CRC cross sections for $1 n$ transfer relative to the first group of states as described above. As can be observed, the description of the data is quite satisfactory.

Concerning the CRC calculations associated with the yields observed in the second group of $1 n$ transfer, $0.80 \leqslant$ $E^{*} \leqslant 2.10 \mathrm{MeV}$, we have assumed a single state to represent all the nearby populated states in ${ }^{119} \mathrm{Sn}$. Within this simplified approach, we have varied the spin, excitation energy, and spectroscopy factor values associated with the coupled state in order to fit the experimental data. As shown in panel (b) of Fig. 9, with $n=2, \ell=2, J=5 / 2^{+}, E^{*}=1.5 \mathrm{MeV}$ and $C^{2} S_{\ell j}=10.2$ we obtained an adequate fit to the data.

TABLE IV. Level structure of ${ }^{119} \mathrm{Sn}$ along with the spectroscopic factors for the overlap $\left\langle{ }^{120} \mathrm{Sn} \mid{ }^{119} \mathrm{Sn}+n\right\rangle$.

\begin{tabular}{lccc}
\hline \hline $\begin{array}{l}E^{*} \\
(\mathrm{keV})\end{array}$ & $\begin{array}{l}\text { State } \\
(n \ell j)\end{array}$ & Parity & $C^{2} S_{\ell j}$ \\
\hline 0.0 & $3 s_{1 / 2}$ & $(+)$ & 0.58 \\
23.8 & $2 d_{3 / 2}$ & $(+)$ & 1.66 \\
89.5 & $1 h_{11 / 2}$ & $(-)$ & 3.35 \\
787.0 & $1 g_{7 / 2}$ & $(+)$ & 5.15 \\
\hline \hline
\end{tabular}

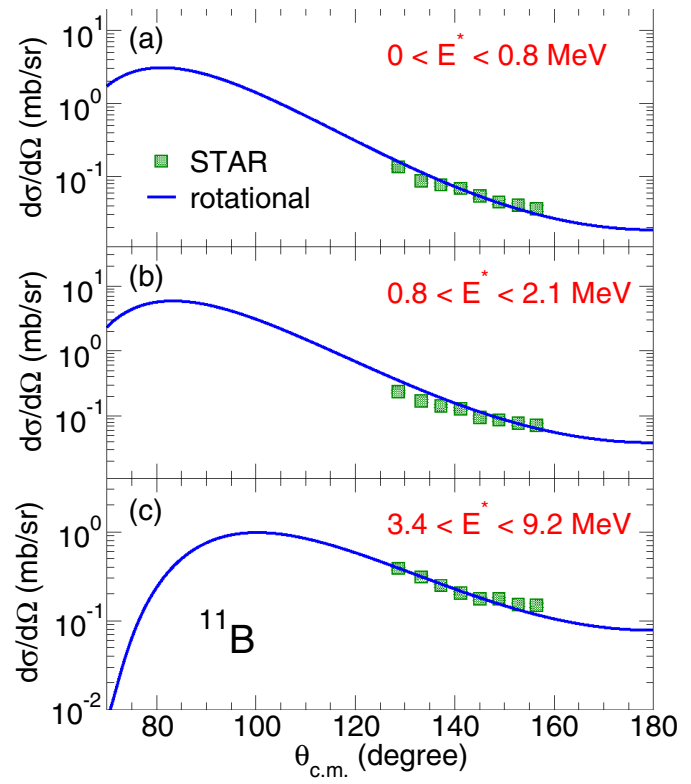

FIG. 9. Experimental cross sections for the $1 n$ transfer channel obtained from the yields associated with the three groups of states in different excitation energy regions of the residual nuclei. The curves represent the results of the CRC calculations (see text for details).

A similar procedure was performed in the case of the third group, $3.4 \leqslant E^{*} \leqslant 9.2 \mathrm{MeV}$. The respective theoretical results shown in panel (c) of Fig. 9 were obtained with $n=2$, $\ell=3, J=5 / 2^{-}, E^{*}=6.0 \mathrm{MeV}$, and $C^{2} S_{\ell j}=9.61$.

In the cases of groups two and three of $1 n$ transfer, there are other possible sets of spin, excitation energy and spectroscopy factor values that provide equally good agreement between data and theory. However, the shape of each theoretical distribution over the entire angular region depends on the choice of these parameters. Therefore, the total (angle integrated) transfer cross section may vary significantly from one choice to another. Our experiment was not particularly optimized for measuring angular distributions for one-nucleon transfer. The STAR telescope was fixed during the experiment and, therefore, the available data only cover a narrow region located at backward angles. Since the transfer angular distributions are peaked in the vicinity of the grazing angle, the theoretical cross sections are much higher around $\theta=80^{\circ}$. This is the reason why our measurements did not determine the total transfer cross sections. We emphasize, however, that, as long as the experimental cross sections for the transfer process are well described by the CRC calculations, the effect of this coupling on the elastic scattering angular distribution (and other channels) is negligible, regardless of the choice of the aforementioned parameters.

\section{One-proton transfer and ${ }^{10} \mathrm{~B} \rightarrow{ }^{1} \mathrm{H}+{ }^{9} \mathrm{Be}$ breakup}

Figure 3 shows that ${ }^{9} \mathrm{Be}$ nuclei have been observed in the $\left(\Delta E, E_{T}\right)$ spectra. In Fig. 10, we present the projection of these events on the $E_{T}$ axis, i.e., the figure represents the number of ${ }^{9} \mathrm{Be}$ events as a function of the total energy of the detected particle. The $E_{\text {Theo }}^{*} \approx 6 \mathrm{MeV}$ for $1 p$ stripping transfer 


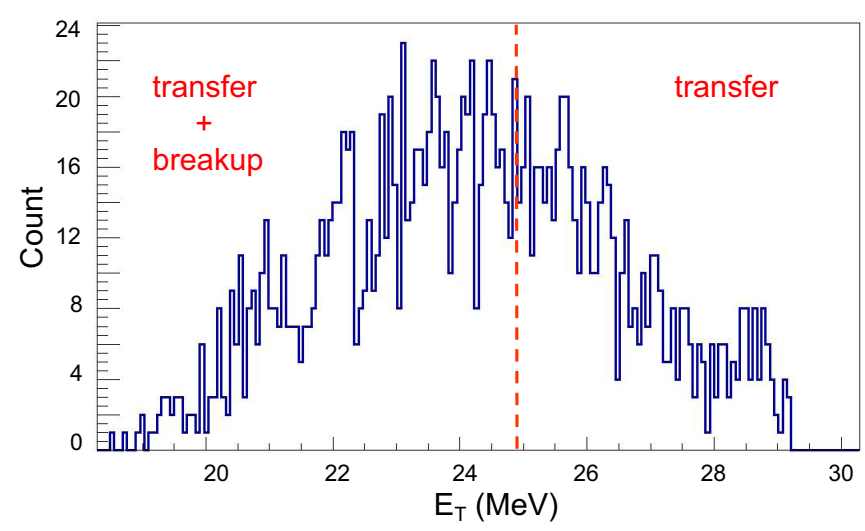

FIG. 10. Energy projection of ${ }^{9} \mathrm{Be}$ nuclei detected at $\theta_{\text {Lab }}=$ $128.6^{\circ}$ with the STAR telescope. The vertical dashed red line separates the regions associated with the breakup and $1 p$ transfer processes.

presented in Table II is in reasonable accordance with the region of highest ${ }^{9} \mathrm{Be}$ yields of Fig. 10: $E_{T}$ (highest yields) $\approx$ $24 \mathrm{MeV} \Longrightarrow E_{\mathrm{Exp}}^{*} \approx 7 \mathrm{MeV}$ (the conversion of $E_{T}$ to $E^{*}$ was performed through the kinematics of the one-proton transfer process). The $1 p$ stripping transfer populating several excited states of the residual nucleus ${ }^{121} \mathrm{Sb}$ has also been reported in Ref. [3] for the ${ }^{7} \mathrm{Li}+{ }^{120} \mathrm{Sn}$ system.

In the present case, we associate these yields with two different reaction processes. Part of the detected ${ }^{9} \mathrm{Be}$ in fact must be associated with the $1 p$ stripping transfer: ${ }^{10} \mathrm{~B}+{ }^{120} \mathrm{Sn} \rightarrow{ }^{9} \mathrm{Be}+{ }^{121} \mathrm{Sb}$. Another fraction should be related to the breakup of ${ }^{10} \mathrm{~B}$ into the fragments ${ }^{1} \mathrm{H}+{ }^{9} \mathrm{Be}$ : ${ }^{10} \mathrm{~B}+{ }^{120} \mathrm{Sn} \rightarrow{ }^{9} \mathrm{Be}+{ }^{1} \mathrm{H}+{ }^{120} \mathrm{Sn}$. To support this statement, it is necessary to introduce arguments related to the energy and kinematics of the detected particles. After calibrating the spectra, it was possible to infer that the detected energies of the ${ }^{9} \mathrm{Be}$ nuclei (for instance, from $E_{T} \approx 19 \mathrm{MeV}$ to $E_{T} \approx 29 \mathrm{MeV}$ in Fig. 10) are associated (from kinematics) with an excitation energy region of $1.0 \leqslant E^{*} \leqslant 12.5 \mathrm{MeV}$. Therefore, the nuclei in the final partition of the one-proton transfer process, ${ }^{9} \mathrm{Be}$, ${ }^{121} \mathrm{Sb}$, or both nuclei, could be found in their excited states, which eventually decay by emitting $\gamma$ rays. On the other hand, the threshold of the binding energy of the ${ }^{121} \mathrm{Sb}$ nucleus $\left({ }^{121} \mathrm{Sb} \rightarrow p+{ }^{120} \mathrm{Sn}\right)$ is $5.8 \mathrm{MeV}$, preventing the occurrence of the breakup process at energies lower than this value. For energies higher than this threshold (indicated by the dashed red line in Fig. 10), the proton can also be transferred to the continuum.

In order to account for the effect of these processes on the elastic channel, we again adopted the simplified approach that considers a proton transfer to a single ${ }^{121} \mathrm{Sb}$ level, with excitation energy lower than the binding energy of ${ }^{1} \mathrm{H}+{ }^{120} \mathrm{Sn}$. The corresponding $1 p$ transfer cross sections obtained within the CRC model are represented by the solid blue line in Fig. 11. We supposed a state with excitation energy of $5.5 \mathrm{MeV}$ for the ${ }^{121} \mathrm{Sb}$ nucleus, with spin $J=1 / 2^{+}, n=3$, and $\ell=0$. In these conditions, we verified that $C^{2} S_{\ell j}=3.24$ gives a satisfactory description of the data (which represent the total yield of ${ }^{9} \mathrm{Be}$ detected). As for the $1 n$ transfer, we have considered a WS

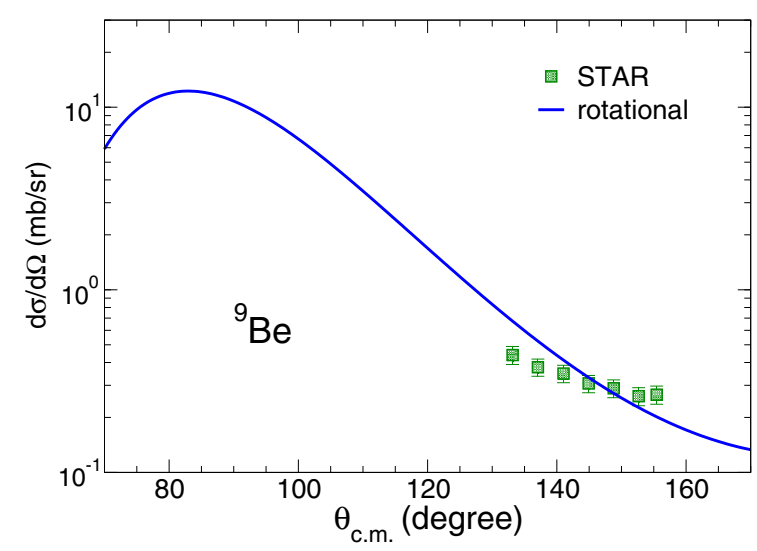

FIG. 11. Experimental angular distribution related to the observed ${ }^{9} \mathrm{Be}$ nuclei. The curve was obtained assuming a schematic CRC calculation of $1 p$ transfer (see text for details).

shape for the proton-core potentials. The parameter values of the WS potentials are given in Table III. As a result of our simulations, we point out that the inclusion of the $1 p$ transfer channel in the coupling scheme also has a negligible effect on the theoretical elastic scattering cross sections.

\section{Other detected transfer and breakup processes}

As shown in Fig. 3, yields of ${ }^{12} \mathrm{C}$ were produced with relatively low statistics from the deuteron pickup transfer reaction ${ }^{120} \mathrm{Sn}\left({ }^{10} \mathrm{~B},{ }^{12} \mathrm{C}\right){ }^{118} \mathrm{In}$. The corresponding experimental cross sections are shown in Fig. 12. A meticulous inspection at Fig. 3 suggests that some ${ }^{11} \mathrm{C}$ nuclei, coming from the proton pickup transfer reaction, could also be present in the $Z=6$ band. However, the contribution of ${ }^{11} \mathrm{C}$ on the total cross section is likely to be very small since this process is hindered by $Q_{\text {opt }}$ considerations (see Table II). The difficulty in separating both nuclei in the mass range of carbon isotopes, associated with the low statistics observed in the spectra, imposes an important experimental challenge. Therefore, the

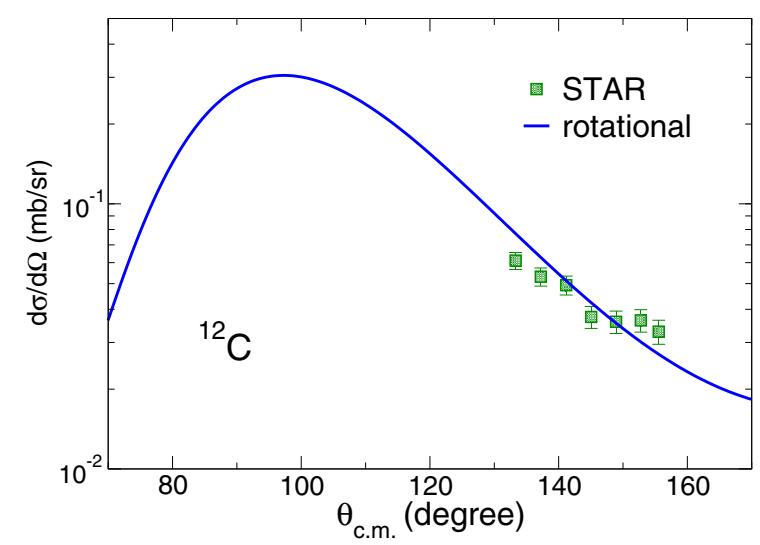

FIG. 12. Experimental angular distribution of ${ }^{12} \mathrm{C}$ nucleus coming from the deuteron pickup transfer reaction. The curve was obtained assuming a schematic CRC calculation of $1 d$ transfer (see text for details). 


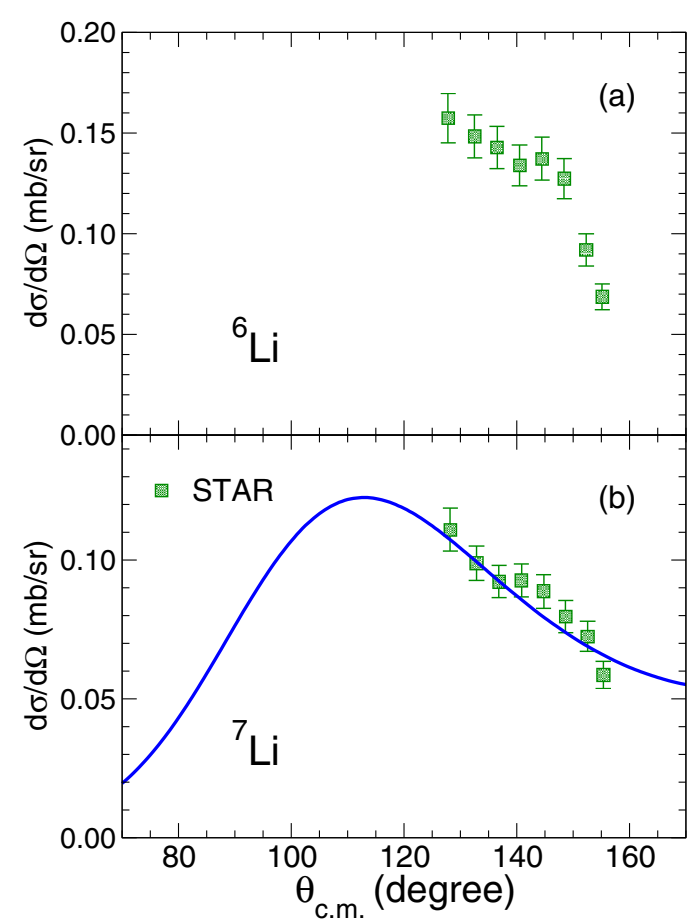

FIG. 13. Panels (a) and (b) present the experimental angular distributions of ${ }^{6} \mathrm{Li}$ and ${ }^{7} \mathrm{Li}$, respectively. The curve presented in panel (b) was obtained assuming a schematic CRC calculation for the ${ }^{3} \mathrm{He}$ transfer. A calculation for the ${ }^{4} \mathrm{He}$ transfer process was not performed in this work (see text for details).

corresponding experimental ${ }^{12} \mathrm{C}$ cross sections might have been slightly overestimated.

We have again used a simplified coupling scheme for this channel, considering the transfer of the deuteron as a cluster through only one state of the ${ }^{121} \mathrm{Sb}$ nucleus. The respective CRC cross sections are shown in Fig. 12 by the solid blue curve. As can be observed in the figure, assuming a single state for the ${ }^{121} \mathrm{Sb}$ nucleus, with an excitation energy of $3.5 \mathrm{MeV}, J=5^{+}, n=4, \ell=4$, and $C^{2} S_{\ell j}=4.41$, we obtained a satisfactory description of the data. The somewhat large value of the spectroscopic factor is acceptable since we are simulating the contribution of a large number of states through a single ${ }^{121} \mathrm{Sb}$ state. The WS parameters assumed for the deuteron-core potentials are given in Table III. This coupling also does not provide a significant contribution to the theoretical elastic scattering cross sections.

Projectile-like fragments with $Z=3$ are clearly observed in the two-dimensional $\left(\Delta E, E_{T}\right)$ spectrum. ${ }^{6} \mathrm{Li}$ has been formed by ${ }^{4} \mathrm{He}$ transfer to unbound states lying in the continuum (breakup), since the threshold energy of the ${ }^{4} \mathrm{He}+{ }^{120} \mathrm{Sn}$ bound system is only $1.852 \mathrm{MeV}$. In fact, the detected energies of ${ }^{6} \mathrm{Li}$ result, through kinematics, in a region of excitation energy of $8.2 \leqslant E^{*} \leqslant 22 \mathrm{MeV}$, with $E_{\text {Exp }}^{*}=12.4 \mathrm{MeV}$ (see Table II). The corresponding experimental cross sections for this process are shown in panel (a) of Fig. 13. The possibility of performing cross section calculations for the $\alpha$ transfer to highly excited unbound states of the final nuclei will not be addressed in this paper.

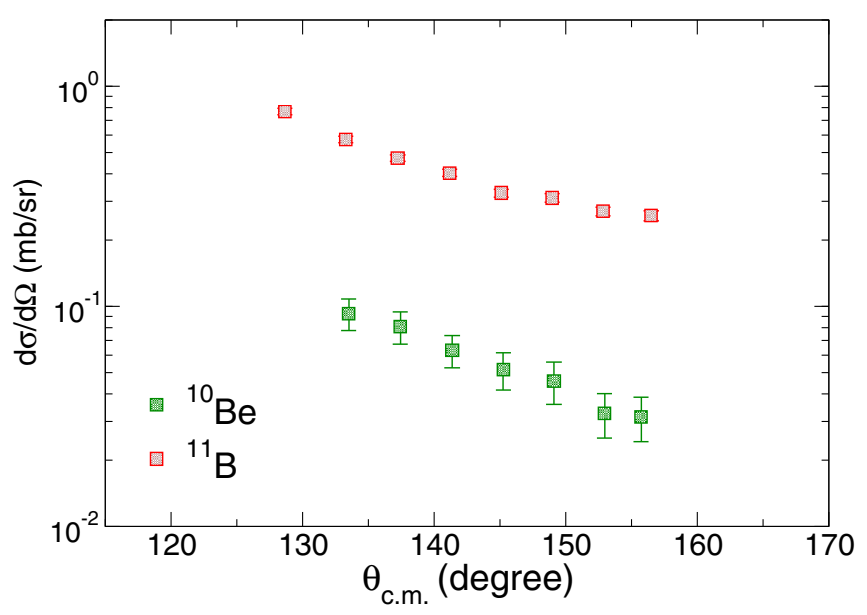

FIG. 14. Experimental angular distribution of ${ }^{10} \mathrm{Be}$ production (green squares). For comparison, the ${ }^{11} \mathrm{~B}$ angular distribution is also shown in the figure.

For the ${ }^{3} \mathrm{He}$ stripping transfer process, ${ }^{120} \mathrm{Sn}\left({ }^{10} \mathrm{~B},{ }^{7} \mathrm{Li}\right){ }^{123} \mathrm{Te}$, the region of detected ${ }^{7} \mathrm{Li}$ events indicates a respective excitation energy region of $8.0 \leqslant E^{*} \leqslant 18.0 \mathrm{MeV}$. As the binding energy for the ${ }^{3} \mathrm{He}+{ }^{120} \mathrm{Sn}$ system is $13.0 \mathrm{MeV}$, a fraction of the transfer cross sections is related to the population of unbound states in the continuum. The data shown in panel (b) of Fig. 13 correspond to the experimental ${ }^{3} \mathrm{He}$ stripping transfer reaction cross sections. Once more, we have adopted a schematic model to simulate the effect of a large number of excited states of the ${ }^{7} \mathrm{Li}$ and ${ }^{123} \mathrm{Te}$ nuclei. The solid blue line in the figure was obtained considering a single state $3 d_{5 / 2}$ of ${ }^{123} \mathrm{Te}$, with $E^{*}=9.2 \mathrm{MeV}$ and $C^{2} S_{\ell j}=19.4$. Again, the high value of this spectroscopic factor is due to the large number of states being represented by this single one. As can be observed, the fit of the data is satisfactory. Table III presents the parameter values of the WS potentials assumed for the ${ }^{3} \mathrm{He}$-core partitions. Most importantly, the effect of this particular coupling on the elastic scattering angular distribution is insignificant.

Figure 5 shows the observed ${ }^{10} \mathrm{Be}$ yields projected on the mass axis obtained using the method described in Ref. [15]. The formation of ${ }^{10} \mathrm{Be}$ nuclei might be associated with the direct SCE, in which the interaction of protons and neutrons can be described in terms of meson exchange. For instance, a proton can become a neutron by emitting a $\pi^{+}$meson. In addition, the observed ${ }^{10} \mathrm{Be}$ can also be related to a two-step transfer process, where a sequential proton pickup followed by a neutron stripping (or vice versa) takes place. Such mechanisms show a strong correlation with the total kinetic energy available in the center-of-mass system [25,26]. At the bombarding energy of the experiment discussed in this paper, it is expected that the contribution of the direct SCE process is significantly smaller than the neutron-proton sequential transfer reaction. The experimental angular distribution obtained for the observed ${ }^{10} \mathrm{Be}$ is presented in Fig. 14. Since it is a two-step process, and the information about the spectroscopic factors of ${ }^{120} \mathrm{Sb}$ is very limited, we do not calculate 


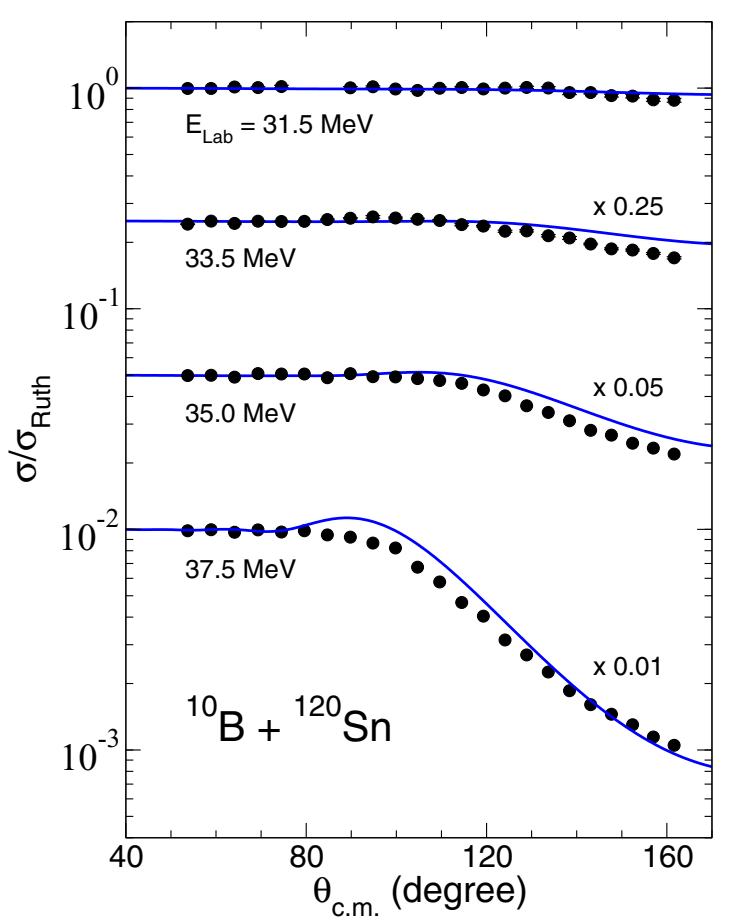

FIG. 15. Experimental elastic scattering angular distribution and corresponding CRC results in several bombarding energies. To avoid superposition of data, some angular distributions have been multiplied by the corresponding values indicated in the figure.

the ${ }^{120} \mathrm{Sn}\left({ }^{10} \mathrm{~B},{ }^{10} \mathrm{Be}\right){ }^{120} \mathrm{Sb}$ cross sections in this paper. On the other hand, the publication of the data is valuable to test theoretical models for SCE reactions at near barrier energies $[27,28]$. Figur 14 also shows the total $1 n$ transfer angular distribution. Clearly, the $1 n$ transfer cross sections leading to the formation of ${ }^{11} \mathrm{~B}$ are significantly larger than the ${ }^{10} \mathrm{Be}$ cross sections, which are possibly related to the two-nucleon transfer of a multistep route involving two successive singlenucleon transfers.

\section{Comparison to other energies}

In previous experimental campaigns carried out at the LAFN, we measured angular distributions of elastic scattering, inelastic excitations and transfer processes for ${ }^{10} \mathrm{~B}+{ }^{120} \mathrm{Sn}$ reaction at four energies spanning the Coulomb barrier: $E_{\mathrm{Lab}}=31.5,33.5,35.0$, and $37.5 \mathrm{MeV}$. In general, the complete data set have been successfully described by CRC calculations within the context of the double-folding SPP. On the occasion, the imaginary part of the optical potential was obtained by multiplying the SPP by the normalization factor of $N_{i}=0.25[10,11]$.

In the present work, we have performed a simultaneous data analyses of all measured energies assuming an internal Woods-Saxon imaginary potential in the CRC calculations. The ${ }^{10} \mathrm{~B}$ was considered a rotor and the s.o. potential was not included in the calculations. The effect of the inelastic couplings on the elastic channel becomes stronger as the bombarding energy increases. As can be noticed in Fig. 15, the agreement between the theoretical curves and the elastic

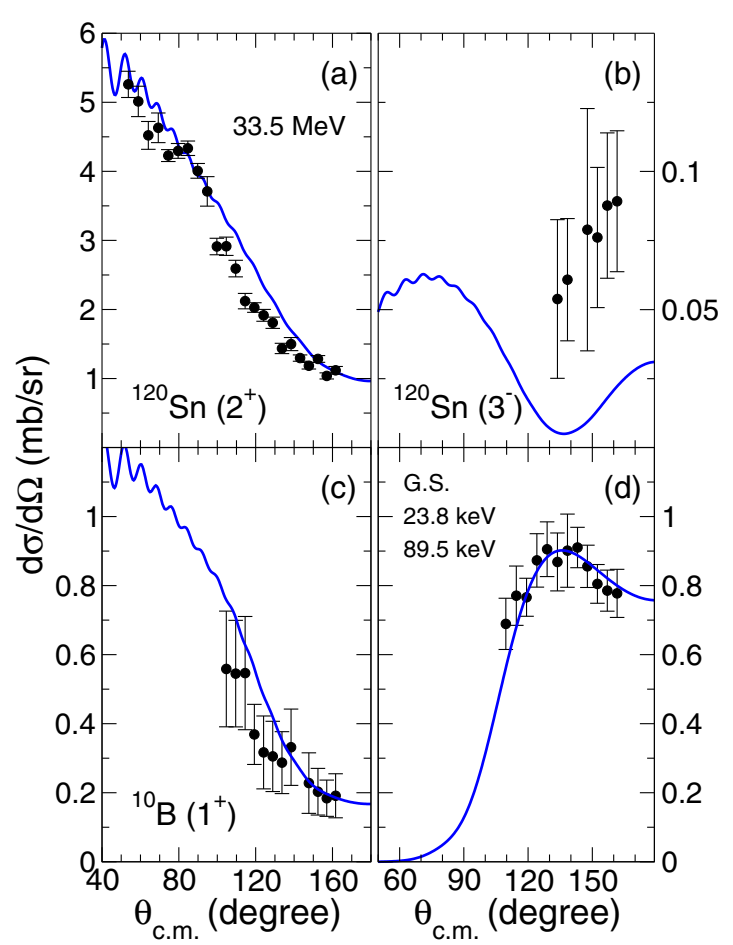

FIG. 16. Inelastic scattering and $1 n$ transfer angular distributions taken at $33.5 \mathrm{MeV}$. The blue solid curves are the results of CRC calculations (see text for details).

scattering data at all previously measured energies is reasonable. As an example, Fig. 16 shows the $33.5 \mathrm{MeV}$ cross sections obtained to the $2_{1}^{+}(1.17 \mathrm{MeV})$ quadrupole and the $3_{1}^{-}$ $(2.40 \mathrm{MeV})$ octupole ${ }^{120} \mathrm{Sn}$ states [panels (a) and (b), respectively], the $1_{1}^{+}(0.72 \mathrm{MeV}){ }^{10} \mathrm{~B}$ quadrupole state in panel (c), and $1 n$ pickup transfer corresponding to the sum of the g.s., $23.8 \mathrm{keV}$, and $89.5 \mathrm{keV}{ }^{119} \mathrm{Sn}$ states in panel (d). Although the description of the data can be improved, especially in the case of panel (b), the overall trend of the angular distributions is well described by the theoretical curves. The results for the other three previously measured energies are quite similar, the $3_{1}^{-}$octupole ${ }^{120} \mathrm{Sn}$ state at $2.40 \mathrm{MeV}$ being the most complicated channel to be properly described by the CRC calculations. Most likely, some channels are still missing in the assumed coupling scheme. Although it is beyond the scope of this work, it would be interesting to perform CDCC calculations considering excited states of the target.

\section{DISCUSSION AND SUMMARY}

Cross sections for elastic, inelastic, and transfer reactions were measured for the ${ }^{10} \mathrm{~B}+{ }^{120} \mathrm{Sn}$ system at $E_{\mathrm{Lab}}=$ $39.70 \mathrm{MeV}$. An overall satisfactory description of the experimental angular distributions was achieved through CRC calculations adopting the SPP as the bare interaction. For the imaginary potential, we assumed a Woods-Saxon shape with a set of parameters resulting only in internal absorption of flux, therefore having a negligible strength at the surface region. The inclusion of a s.o. potential in the central interaction does not significantly affect the data fit. For consistency, the 
parameter values of the s.o. potential assumed here are the same as those obtained in the data analyses of the ${ }^{10} \mathrm{~B}+{ }^{197} \mathrm{Au}$ system $[13,14]$. In that case, the effect of the s.o. potential on the cross sections is important only at quite high energies, a result consistent with that obtained in the present work.

In the case of the elastic and inelastic scattering, the agreement between data and theory is somewhat better in the case where the ${ }^{10} \mathrm{~B}$ nucleus is treated as a rotor, showing that the $\mathrm{CRC}$ results present a moderate dependence on the assumption for the collective model of the highly deformed ${ }^{10} \mathrm{~B}$.

The experimental cross sections for several processes involve the contribution of many states with high excitation energies. In these cases, we have adopted the procedure of including only one state (for each process) in the CRC calculations, with parameter values adjusted to fit the data. The main purpose of this procedure is to evaluate the effect of these couplings on the elastic scattering cross section as well as on the cross sections of the other reaction channels. Among all channels included in the CRC calculations, only the couplings related to inelastic excitation play a significant role, resulting in a large effect on the elastic scattering, the main contribution being that from the first ${ }^{10} \mathrm{~B}$ excited state. Even though the effect of the couplings on the elastic channel becomes more relevant as the bombarding energy increases, a similar behavior was noticed for the other lower energies previously measured at LAFN $[10,11]$.

Although the effect of the couplings on the elastic channel is strong, the cross sections obtained with the full CRC calculations slightly underpredict the data. The discrepancy becomes more relevant as the scattering angle increases. It is possible to obtain a better data description by including a normalization of the SPP by a factor 0.88 . Another possible source for this discrepancy could be related to the effect of couplings not included in the present CRC calculations. The overall results of a simultaneous data analyses of all measured energies, considering an internal Woods-Saxon imaginary optical potential, also corroborate this statement. In fact, quite similar behavior of the elastic scattering cross sections has been observed for many $\alpha$-nucleus systems and might be related to the effect of couplings to inelastic states of very high excitation energies (even with negligible cross sections [29-33]. In addition, it would be interesting to investigate the effect of the breakup processes on the elastic scattering channel, possibly taking into account the excitation of the target.

In our experiment, we have observed ${ }^{10} \mathrm{Be}$ yields, which are likely to be related to a two-step transfer process, where a sequential proton pickup followed by a neutron stripping (or vice versa) takes place. In addition, we have detected ${ }^{9} \mathrm{Be},{ }^{6} \mathrm{Li}$, and ${ }^{7} \mathrm{Li}$ nuclei that are associated to the breakup processes: ${ }^{10} \mathrm{~B} \rightarrow{ }^{1} \mathrm{H}+{ }^{9} \mathrm{Be},{ }^{10} \mathrm{~B} \rightarrow{ }^{4} \mathrm{He}+{ }^{6} \mathrm{Li}$ and ${ }^{10} \mathrm{~B} \rightarrow{ }^{3} \mathrm{He}+{ }^{7} \mathrm{Li}$. It is interesting to note that ${ }^{9} \mathrm{Be}$ was also detected in the measurements of the ${ }^{10} \mathrm{~B}+{ }^{197} \mathrm{Au}$ system at low energies, but ${ }^{6} \mathrm{Li}$ and ${ }^{7} \mathrm{Li}$ were not observed [13,14]. A consistent data analyses for both systems, including these breakup processes through CDCC calculations would be interesting, although this task is out of the scope of the present paper.

\section{ACKNOWLEDGMENTS}

This work was partially supported by Fundação de Amparo à Pesquisa do Estado de São Paulo (FAPESP) Proc. No. 2018/09998-8, No. 2019/07767-1, No. 2019/05769-7, and No. 2017/05660-0; by Conselho Nacional de Desenvolvimento Científico e Tecnológico (CNPq) Proc. No. 304056/2019-7, No. 302160/2018-3, and No. 306433/20176; and by and project INCT-FNA Proc. No 464898/2014-5. M.A.G.A. and J.P.F.-G. acknowledge support from the Ministry of Science, Innovation and Universities of Spain, through the project PGC2018-096994-B-C21. M.R.-G. acknowledges support by the Spanish Ministerio de Economía y Competitividad and FEDER funds under Project No. FIS2017-88410-P.
[1] L. R. Gasques, D. J. Hinde, M. Dasgupta, A. Mukherjee, and R. G. Thomas, Phys. Rev. C 79, 034605 (2009).

[2] A. Shrivastava et al., Phys. Lett. B 718, 931 (2013).

[3] A. Kundu, S. Santra, A. Pal, D. Chattopadhyay, R. Tripathi, B. J. Roy, T. N. Nag, B. K. Nayak, A. Saxena, and S. Kailas, Phys. Rev. C 95, 034615 (2017).

[4] A. Diaz-Torres, D. J. Hinde, J. A. Tostevin, M. Dasgupta, and L. R. Gasques, Phys. Rev. Lett. 98, 152701 (2007).

[5] S. Kalkal, E. C. Simpson, D. H. Luong, K. J. Cook, M. Dasgupta, D. J. Hinde, I. P. Carter, D. Y. Jeung, G. Mohanto, C. S. Palshetkar, E. Prasad et al., Phys. Rev. C 93, 044605 (2016).

[6] L. F. Canto, P. R. S. Gomes, R. Donangelo, and M. S. Hussein, Phys. Rep. 424, 1 (2006).

[7] N. Keeley, N. Alamanos, K. W. Kemper, and K. Rusek, Part. Nucl. Phys. 63, 396 (2009).

[8] L. F. Canto, P. R. S. Gomes, R. Donangelo, J. Lubian, and M. S. Hussein, Phys. Rep. 596, 1 (2015).
[9] V. A. B. Zagatto, J. Lubian, L. R. Gasques, M. A. G. Alvarez, L. C. Chamon, J. R. B. Oliveira, J. A. Alcantara-Nunez, N. H. Medina, V. Scarduelli, A. Freitas, I. Padron, E. S. Rossi, and J. M. B. Shorto, Phys. Rev. C 95, 064614 (2017).

[10] L. R. Gasques et al., Phys. Rev. C 97, 034629 (2018).

[11] M. A. G. Alvarez et al., Phys. Rev. C 98, 024621 (2018).

[12] L. C. Chamon et al., Phys. Rev. C 66, 014610 (2002).

[13] M. Aversa et al., Phys. Rev. C 101, 044601 (2020).

[14] L. R. Gasques et al., Phys. Rev. C 101, 044604 (2020).

[15] V. Scarduelli, L. R. Gasques, L. C. Chamon, and A. LépineSzily, Eur. Phys. J. A 56, 24 (2020).

[16] I. J. Thompson, Comput. Phys. Rep. 7, 167 (1988).

[17] D. F. M. Botero, L. C. Chamon, and B. V. Carlson, J. Phys. G 44, 105102 (2017).

[18] L. C. Chamon and B. V. Carlson, Nucl. Phys. A 846, 1 (2010).

[19] D. G. Alkhazov, Y. P. Gangrskii, I. K. Lemberg, and Y. I. Udralov, Bull. Acad. Sci USSR (Phys. Ser.) 28, 149 (1965). 
[20] N. G. Jonsson, A. Backlin, J. Kantele, R. Julin, M. Lountama, and A. Passoja, Nucl. Phys. A 371, 333 (1981).

[21] O. Beer, A. E. Behay, P. Lopato, Y. Terrien, G. Vallois, and K. K. Seth, Nucl. Phys. A 147, 326 (1970).

[22] G. Bruge, J. Faivre, H. Faraggi, and A. Bussiere, Nucl. Phys. A 146, 597 (1970).

[23] T. Kibédi and R. H. Spear, At. Data Nucl. Data Tables 80, 35 (2002).

[24] R. Broglia and A. Winther, Heavy Ion Reactions: Lecture Notes the Elementary Processes, Frontiers in Physics Vol. 84 (Addison-Wesley, Redwood City, CA, 1991).

[25] H. G. Bohlen, B. Gebauer, D. Kolbert, S. Kubono, W. von Oertzen, P. O. Pellegrin, E. Stiliaris, M. Wilpert, T. Wilpert, H. Lenske, H. H. Wolter et al., Nucl. Phys. A 488, 89c (1988).
[26] H. Lenske, H. H. Wolter, and H. G. Bohlen, Phys. Rev. Lett. 62, 1457 (1989).

[27] B. M. Loc, N. Auerbach, and D. T. Khoa, Phys. Rev. C 96, 014311 (2017).

[28] H. Lenske, J. I. Bellone, M. Colonna, and J. A. Lay, Phys. Rev. C 98, 044620 (2018).

[29] M. Evers, M. Dasgupta, D. J. Hinde, L. R. Gasques, M. L. Brown, R. Rafiei, and R. G. Thomas, Phys. Rev. C 78, 034614 (2008).

[30] L. C. Chamon, L. R. Gasques, G. P. A. Nobre, E. S. Rossi, Jr, R. J. deBoer, C. Seymour, M. Wiescher, and G. G. Kiss, J. Phys. G 42, 055102 (2015).

[31] L. C. Chamon and L. R. Gasques, J. Phys. G 43, 015107 (2016).

[32] L. C. Chamon, L. R. Gasques, and J. C. Zamora, J. Phys. G 47, 105103 (2020).

[33] L. R. Gasques et al., Phys. Rev. C 76, 024612 (2007). 